\title{
A Bottom-Up Dynamic Model of Portfolio Credit Risk. Part I: Markov Copula Perspective
}

\author{
Tomasz R. Bielecki ${ }^{1, *}$, Areski Cousin ${ }^{2 \dagger}$, \\ Stéphane Crépey ${ }^{3, \ddagger}, \quad$ Alexander Herbertsson ${ }^{4, \S}$ \\ ${ }^{1}$ Department of Applied Mathematics \\ Illinois Institute of Technology, Chicago, IL 60616, USA \\ ${ }^{2}$ Université de Lyon, Université Lyon 1, LSAF, France \\ ${ }^{3}$ Laboratoire Analyse et Probabilités \\ Université d'Évry Val d'Essonne, 91037 Évry Cedex, France \\ ${ }^{4}$ Centre for finance/Department of Economics \\ University of Gothenburg, SE 40530 Göteborg, Sweden
}

March 8, 2013

\begin{abstract}
We consider a bottom-up Markovian copula model of portfolio credit risk where instantaneous contagion is possible in the form of simultaneous defaults. Due to the Markovian copula nature of the model, calibration of marginals and dependence parameters can be performed separately using a two-steps procedure, much like in a standard static copula set-up. In this sense this model solves the bottom-up top-down puzzle which the CDO industry had been trying to do for a long time. It can be applied to any dynamic credit issue like consistent valuation and hedging of CDSs, CDOs and counterparty risk on credit portfolios.
\end{abstract}

Keywords: Portfolio credit risk, Credit derivatives, Markov copula model, Common shocks, Dynamic hedging.

\footnotetext{
${ }^{*}$ The research of T.R. Bielecki was supported by NSF Grant DMS-0604789 and NSF Grant DMS-0908099.

${ }^{\dagger}$ The research of A. Cousin benefited from the support of the DGE and the ANR project Ast\&Risk.

${ }^{\ddagger}$ The research of S. Crépey benefited from the support of the 'Chaire Risque de crédit' and of the "Chaire Marchés en Mutation", Fédération Bancaire Française.

${ }^{\S}$ The research of A. Herbertsson was supported by the Jan Wallander and Tom Hedelius Foundation and by Vinnova
} 


\section{Introduction}

The CDO market have been deeply and adversely impacted by the crisis. In particular, CDO issuances have become quite rare. Nevertheless, there are huge notionals of CDO contracts outstanding and market participants continue to be confronted with the task to hedge their positions in these contracts up to maturity date. Moreover, according to the regulation (see [2]), tranches on standard indices and their associated liquid hedging positions continue to be charged as hedge-sets under internal VaR-based method. About the CDO hedging issue we refer the reader to Laurent, Cousin and Fermanian [28], Frey and Backhaus [23], Cont and Kan [15] or Cousin, Crépey and Kan [17]. In particular it has been established empirically in [15] and [17] that a single-instrument hedge of a CDO tranche by the corresponding credit index is often not good enough. In this and the companion paper [5], we deal with a bottom-up Markovian copula model, in which hedging loss derivatives by single-name instruments can be performed in a theoretically sound and practical way. There are two major theoretical contributions of these papers:

- In this paper, we construct a Markov model where dependence between default risks derives from the possibility of joint defaults. The Markovian structure of the model is adequate for the problem at hand, that is for the problem of dynamic hedging of portfolio credit risk. The (dynamic) copula property of the model allows for separation of calibration of the univariate marginals of the underlying multivariate Markov process, from calibration of the dependence structure between the components of the process. This is of critical importance from the practical point of view.

- We show that the conditional dependence structure of default times belongs to the class of Marshall-Olkin copulas (see Prop 2.9]). This result is exploited in [5] to construct an equivalent conditional factor representation of our Markovian model which relies on "common shocks", the latter being represented by Cox processes likely to trigger defaults simultaneously in some pre-specifed group of obligors. This is important from the practical point of view as this interpretation underlies semi-explicit convolution-based pricing schemes to assess the credit portfolio loss distribution at several time horizons. Such numerical schemes play a crucial role when calibrating credit portfolio models and in related applications such as hedging portfolio credit derivatives by individual names, or counterparty risk valuation for portfolios (see [1, 7]).

The common shock aspect of our model is related to the work by Elouerkhaoui [21] (see also Brigo et al. [12, 13, 14]). Consequently, some results derived in this and the companion paper are consistent with results derived in [21]. However, there are major differences between our study and the one presented in [21]:

- Firstly, the approach of [21] suffers from the "curse of dimensionality" due to the need of summation (integration) over the set denoted by $\Pi_{n}$ in [21] (see for example equation (2.6) therein, and compare with our own result (17) below), the set of all subsets of the set $\{1,2, \ldots, n\}$. By contrast, the complexity of our formula (7) for the generator of our Markov process, or of our common shock algorithms described in Subsection 2.1 of the companion paper [5], are controlled by the cardinality of our shocks set $\mathcal{Y}$, typically a few units in applications (see [5]).

- Secondly, as already stated, our methodology allows for separation of calibration of idiosyncratic (marginal) laws of the underlying Markov process, from the calibration of 
the dependence structure of the process. The calibration really amounts to calibrating the infinitesimal generator of the underlying Markov process, and once this is done, the model can be used for consistent pricing and hedging of both the underlying products, such as CDO tranches, as well as options on such with future expiration dates (e.g. in the context of CVA computations); this feature obviously contributes to increased practical use of our methodology. In this sense, our Markov copula model is a genuine dynamic model, as a model of dependence between underlying stochastic processes. This not really the case with the model developed in [21, where the "dynamic copula" feature is in the sense of Patton's conditional copula [31], which is a stochastic process itself, and as such can't be calibrated to initial data.

- Last, but not least, the Markov copula approach of this paper is generic in the sense that, as demonstrated in [10, 11, it also applies to modeling of dynamics of credit ratings. This is not the case with the approach of [21].

Comparing now our methodology to the what is done in Brigo et al. [12, 13, 14, we see that the major differences can be summarized as follows:

- Our approach is a bottom-up approach, hence an approach applicable for hedging basket products using individual names, whereas the approach taken in [12, 13] is a top-down approach, and, as such, is not applicable for hedging basket products using individual names;

- This also applies to the so-called GPCL extension of the model of [14] in which individual names are represented of the model so that, in principle, hedging basket products using individual names could be considered in this setup. This is not practical however because fault of a suitable decoupling property between the dependence structure and the individual names in the model, the calibration of the model can only be addressed through a global joint optimization procedures involving all the model parameters at the same time, which is untractable numerically.

- Again, our approach is generic in the sense that it also applies to modeling of dynamics of credit ratings. This is not the case with the approach of [12, 13, 14].

This paper is organized as follows. In Section 2 we formulate a bottom-up Markovian copula model, in which individual default processes for various credit names are coupled together by means of simultaneous defaults. In Section 3 we exploit the dynamic structure of the model to derive explicit dynamic min-variance hedging formulas. The more technical proofs are deferred to Appendix A. The algorithmic aspects of the model based on the common-shock representation, as well as illustrative numerics, are provided in the companion paper [5]. Fine features of the modeling of the default marginals (single-name modeling in different kinds of affine setups) are considered in [6]. A short announcing version of these results can be found in [4].

In the rest of the paper we consider a risk neutral pricing model $(\Omega, \mathcal{F}, \mathbb{P})$, for a filtration $\mathcal{F}=\left(\mathcal{F}_{t}\right)_{t \in[0, T]}$ which will be specified below and where $T \geq 0$ is a fixed time horizon. We denote $N_{n}=\{1, \ldots, n\}$ and let $\mathcal{N}_{n}$ denote the set of all subsets of $N_{n}$ where $n$ represents the number of obligors in the underlying credit portfolio. Further, we set $\max \emptyset=-\infty$. 


\section{Model of Default Times}

In this section we construct a bottom-up Markovian model consisting of a multivariate factor process $\mathbf{X}$ and a vector $\mathbf{H}$ representing the default indicator processes in a pool of $n$ different credit names. More specifically, $\mathbf{H}_{t}$ is a vector in $\{0,1\}^{n}$ where the $i$-th entry of $\mathbf{H}_{t}$ is the indicator function for the event of a default of obligor $i$ up to time $t$. The purpose of the factor process $\mathbf{X}$ is to more realistically model diffusive randomness of credit spreads.

In our model, defaults are the consequence of some "shocks" associated with groups of obligors. We define the following pre-specified set of groups

$$
\mathcal{Y}=\left\{\{1\}, \ldots,\{n\}, I_{1}, \ldots, I_{m}\right\},
$$

where $I_{1}, \ldots, I_{m}$ are subsets of $\{1, \ldots, n\}$, and each group $I_{j}$ contains at least two obligors or more. The shocks are divided in two categories: the "idiosyncratic" shocks associated with singletons $\{1\}, \ldots,\{n\}$ can only trigger the default of name $1, \ldots, n$ individually, while the "systemic" shocks associated with multi-name groups $I_{1}, \ldots, I_{m}$ may simultaneously trigger the default of all names in these groups. Note that several groups $I_{j}$ may contain a given name $i$, so that only the shock occurring first effectively triggers the default of that name. As a result, when a shock associated with a specific group occurs at time $t$, it only triggers the default of names that are still alive in that group at time $t$. In the following, the elements $Y$ of $\mathcal{Y}$ will be used to designate shocks and we let $\mathcal{I}=\left(I_{l}\right)_{1 \leq l \leq m}$ denote the pre-specified set of multi-name groups of obligors.

Let $\nu=|\mathcal{Y}|=n+m$ denote the cardinality of $\mathcal{Y}$. Given a multivariate Brownian motion $\mathbf{W}=\left(W^{Y}\right)_{Y \in \mathcal{Y}}$ with independent components, we assume that the factor process $\mathbf{X}=\left(X^{Y}\right)_{Y \in \mathcal{Y}}$ is a strong solution to

$$
d X_{t}^{Y}=b_{Y}\left(t, X_{t}^{Y}\right) d t+\sigma_{Y}\left(t, X_{t}^{Y}\right) d W_{t}^{Y}
$$

for suitable drift and diffusion functions $b_{Y}=b_{Y}(t, x)$ and $\sigma_{Y}=\sigma_{Y}(t, x)$. By application of Theorem 32 page 100 of Protter [32], this makes $\mathbf{X}$ an $\mathcal{F}^{\mathbf{W}}$-Markov process admitting the following generator acting on functions $v=v(t, \mathbf{x})$ with $\mathbf{x}=\left(x_{Y}\right)_{Y \in \mathcal{Y}}$

$$
A_{t} v(t, \mathbf{x})=\sum_{Y \in \mathcal{Y}}\left(b_{Y}\left(t, x_{Y}\right) \partial_{x_{Y}} v(t, \mathbf{x})+\frac{1}{2} \sigma_{Y}^{2}\left(t, x_{Y}\right) \partial_{x_{Y}^{2}}^{2} v(t, \mathbf{x})\right)
$$

Let $\mathcal{F}:=\mathcal{F}^{(\mathbf{W}, \mathbf{H})}$ be the filtration generated by the Brownian motion $\mathbf{W}$ and the point process $\mathbf{H}$. Given the "intensity functions" of shocks, say $\lambda_{Y}=\lambda_{Y}\left(t, x_{Y}\right)$ for every shock $Y \in \mathcal{Y}$, we would like to construct a model in which the $\mathcal{F}$-predictable intensity of a jump of $\mathbf{H}=\left(H^{i}\right)_{1 \leq i \leq n}$ from $\mathbf{H}_{t-}=\mathbf{k}$ to $\mathbf{H}_{t}=\mathbf{l}$, with $\mathbf{l} \neq \mathbf{k}$ in $\{0,1\}^{n}$, is given by

$$
\lambda\left(t, \mathbf{X}_{t}, \mathbf{k}, \mathbf{l}\right):=\sum_{\left\{Y \in \mathcal{Y} ; \mathbf{k}^{Y}=\mathbf{l}\right\}} \lambda_{Y}\left(t, X_{t}^{Y}\right),
$$

where, for any $Z \in \mathcal{N}_{n}$, the expression $\mathbf{k}^{Z}$ denotes the vector obtained from $\mathbf{k}=\left(k_{1}, \ldots, k_{n}\right)$ by replacing the components $k_{i}, i \in Z$, by numbers one (whenever $k_{i}$ is not equal to one already). The intensity of a jump of $\mathbf{H}$ from $\mathbf{k}$ to $\mathbf{l}$ at time $t$ is thus equal to the sum of the intensities of the shocks $Y \in \mathcal{Y}$ such that, if the joint default of the survivors in group $Y$ occurred at time $t$, then the state of $\mathbf{H}$ would move from $\mathbf{k}$ to $\mathbf{l}$.

Example 2.1 Figure 1 shows one possible defaults path in our model with $n=5$ and $\mathcal{Y}=\{\{1\},\{2\},\{3\},\{4\},\{5\},\{4,5\},\{2,3,4\},\{1,2\}\}$. The inner oval shows which commonshock happened and caused the observed default scenarios at successive default times. At 
the first instant, default of name 2 is observed as the consequence of the idiosyncratic shock $\{2\}$. At the second instant, names 4 and 5 have defaulted simultaneously as a consequence of the systemic shock $\{4,5\}$. At the fourth instant, the systemic shock $\{2,3,4\}$ triggers the default of name 3 alone as name 2 and 4 have already defaulted. At the fifth instant, default of name 1 alone is observed as the consequence of the systemic shock $\{1,2\}$. Note that the information produced by the arrival of the shock-events cannot be deduced from the mere observation of the sequence of states followed by $\mathbf{H}_{t}$.

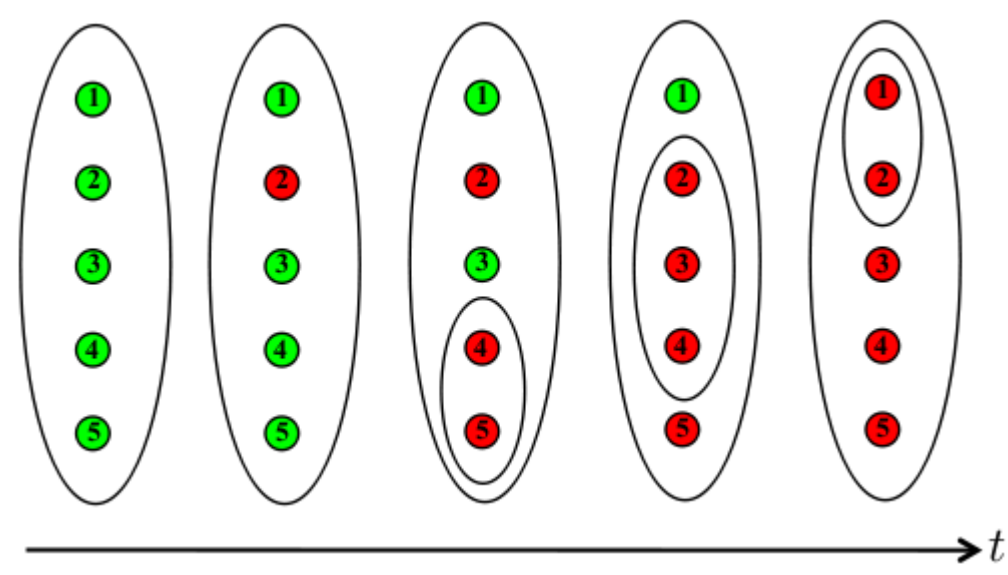

Figure 1: One possible defaults path in a model with $n=5$ and $\mathcal{Y}=$ $\{\{1\},\{2\},\{3\},\{4\},\{5\},\{4,5\},\{2,3,4\},\{1,2\}\}$.

To achieve (3) we follow the classical methodology: we construct $\mathbf{H}$ by an $\mathbf{X}$-related change of probability measure, starting from a continuous-time Markov chain with intensity one. This construction is detailed in Appendix A.1.

\subsection{Itô Formula}

In this subsection we state the Itô formula for functions of the Markov process $(\mathbf{X}, \mathbf{H})$.

For any set $Z \in \mathcal{N}_{n}$, let the set-event indicator process $H^{Z}$ denote the indicator process of a joint default of the names in $Z$ and only in $Z$. For $\mathbf{k}=\left(k_{1}, \ldots, k_{n}\right) \in\{0,1\}^{n}$, we introduce $\operatorname{supp}(\mathbf{k})=\left\{i \in N_{n} ; k_{i}=1\right\}$ and $\operatorname{supp}^{c}(\mathbf{k})=\left\{i \in N_{n} ; k_{i}=0\right\}$. Hence, $\operatorname{supp}(\mathbf{k})$ denotes the obligors who have defaulted in state $\mathbf{k}$ and similarly $\operatorname{supp}^{c}(\mathbf{k})$ are the survived names in the portfolio-state $\mathbf{k}$.

The following lemma provides the structure of the so called compensated set-event martingales $M^{Z}$, which we will use later as fundamental martingales to represent the pure jump martingale components of the various price processes involved.

Lemma 2.2 For every set $Z \in \mathcal{N}_{n}$ the intensity of $H^{Z}$ is given by $\ell_{Z}\left(t, \mathbf{X}_{t}, \mathbf{H}_{t}\right)$, so

$$
d M_{t}^{Z}=d H_{t}^{Z}-\ell_{Z}\left(t, \mathbf{X}_{t}, \mathbf{H}_{t}\right) d t
$$

is a martingale, and the set-event intensity function $\ell_{Z}(t, \mathbf{x}, \mathbf{k})$ is defined as

$$
\ell_{Z}(t, \mathbf{x}, \mathbf{k})=\sum_{Y \in \mathcal{Y} ; Y \cap \operatorname{supp}^{c}(\mathbf{k})=Z} \lambda_{Y}\left(t, x_{Y}\right)
$$


Proof. See Appendix A.1.1.

So $\ell_{Z}\left(t, \mathbf{X}_{t}, \mathbf{H}_{t-}\right)=\sum_{Y \in \mathcal{Y} ; Y_{t}=Z} \lambda_{Y}\left(t, X_{t}^{Y}\right)$, where for every $Y$ in $\mathcal{Y}=\left\{\{1\}, \ldots,\{n\}, I_{1}, \ldots, I_{m}\right\}$ we define

$$
Y_{t}=Y \cap \operatorname{supp}^{c}\left(\mathbf{H}_{t-}\right),
$$

the set-valued process representing the survived obligors in $Y$ right before time $t$. Let also $\mathcal{Z}_{t}=\left\{Z \in N_{n} ; Z=Y_{t}\right.$ for at least one $\left.Y \in \mathcal{Y}\right\} \backslash \emptyset$ denote the set of all non-empty sets of survivors of sets $Y$ in $\mathcal{Y}$ right before time $t$.

We now derive a version of the Itô formula, which is relevant for our model. It will be used below for establishing the Markov properties of our set-up, as well as for deriving price dynamics. Let $\sigma(t, \mathbf{x})$ denote the diagonal matrix with diagonal $\left(\sigma_{Y}\left(t, x_{Y}\right)\right)_{Y \in \mathcal{Y}}$. Given a function $u=u(t, \mathbf{x}, \mathbf{k})$ with $\mathbf{x}=\left(x_{Y}\right)_{Y \in \mathcal{Y}}$ and $\mathbf{k}=\left(k_{i}\right)_{1 \leq i \leq n}$ in $\{0,1\}^{n}$, we denote $\nabla u(t, \mathbf{x}, \mathbf{k})=\left(\partial_{x_{Y}} u(t, \mathbf{x}, \mathbf{k}), Y \in \mathcal{Y}\right)$, the (row-)gradient of $u$ with respect to $\mathbf{x}$. Let also $\delta u^{Z}$ represent the sensitivity of $u$ to the event $Z \in \mathcal{N}_{n}$, so

$$
\delta u^{Z}(t, \mathbf{x}, \mathbf{k})=u\left(t, \mathbf{x}, \mathbf{k}^{Z}\right)-u(t, \mathbf{x}, \mathbf{k}) .
$$

Proposition 2.3 Given a regular enough function $u=u(t, \mathbf{x}, \mathbf{k})$, one has

$$
\begin{gathered}
d u\left(t, \mathbf{X}_{t}, \mathbf{H}_{t}\right)=\left(\partial_{t}+\mathcal{A}_{t}\right) u\left(t, \mathbf{X}_{t}, \mathbf{H}_{t}\right) d t+\nabla u\left(t, \mathbf{X}_{t}, \mathbf{H}_{t}\right) \sigma\left(t, \mathbf{X}_{t}\right) d \mathbf{W}_{t} \\
+\sum_{Z \in \mathcal{Z}_{t}} \delta u^{Z}\left(t, \mathbf{X}_{t}, \mathbf{H}_{t-}\right) d M_{t}^{Z}
\end{gathered}
$$

where

$$
\begin{aligned}
\mathcal{A}_{t} u(t, \mathbf{x}, \mathbf{k})= & \sum_{Y \in \mathcal{Y}}\left(b_{Y}\left(t, x_{Y}\right) \partial_{x_{Y}} u(t, \mathbf{x}, \mathbf{k})+\frac{1}{2} \sigma_{Y}^{2}\left(t, x_{Y}\right) \partial_{x_{Y}^{2}}^{2} u(t, \mathbf{x}, \mathbf{k})\right) \\
& +\sum_{Y \in \mathcal{Y}} \lambda_{Y}\left(t, x_{Y}\right) \delta u^{Y}(t, \mathbf{x}, \mathbf{k}) .
\end{aligned}
$$

Proof. See Appendix A.1.2.

In the Itô formula (6), the jump term may involve any of the $2^{n}$ set-events martingales $M^{Z}$ for $Z \in \mathcal{N}_{n}$. This suggests that the martingale dimension ${ }^{1}$ of the model is $\nu+2^{n}$, where $\nu=n+m$ corresponds to the dimension of the Brownian motion $\mathbf{W}$ driving the factor process $\mathbf{X}$ and $2^{n}$ corresponds to the jump component $\mathbf{H}$. Yet by a reduction which is due to specific structure of the intensities in our set-up, the jump term of $\mathcal{A}_{t}$ in (7) is a sum over the set of shocks $\mathcal{Y}$, which has cardinality $\nu$.

Note that our model excludes direct contagion effects in which intensities of surviving names would be affected by past defaults, as opposed to the bottom-up contagion models treated by e.g. [16, 24, 25, 28]. To provide some understanding in this regard, we give a simple illustrative example.

\footnotetext{
${ }^{1}$ Minimal number of fundamental martingales which can be used as integrators to represent all the martingales in the model, see Appendix A.1.
} 
Example 2.4 Take $N_{n}=\{1,2,3\}$, so that the state space of $\mathbf{H}$ contains 8 elements:

$$
\{(0,0,0),(1,0,0),(0,1,0),(0,0,1),(1,1,0),(1,0,1),(0,1,1),(1,1,1)\} .
$$

Now, let $\mathcal{Y}$ be given as $\mathcal{Y}=\{\{1\},\{2\},\{3\},\{1,2\},\{1,2,3\}\}$. This is an example of the nested structure of $\mathcal{I}$ with $I_{1}=\{1,2\} \subset I_{2}=\{1,2,3\}$. Suppose for simplicity that $\lambda_{Y}$ does not depend either on $t$ or on $\mathbf{x}$ (dependence in $t, \mathbf{x}$ will be dealt with in Subsection 2.2). Then, the generator $\mathcal{A}$ of the chain $\mathbf{H}$ is given in matrix-form by

$$
\mathcal{A} \equiv\left[\begin{array}{cccccccc}
\cdot & \lambda_{\{1\}} & \lambda_{\{2\}} & \lambda_{\{3\}} & \lambda_{\{1,2\}} & 0 & 0 & \lambda_{\{1,2,3\}} \\
0 & \cdot & 0 & 0 & \lambda_{\{2\}}+\lambda_{\{1,2\}} & \lambda_{\{3\}} & 0 & \lambda_{\{1,2,3\}} \\
0 & 0 & \cdot & 0 & \lambda_{\{1\}}+\lambda_{\{1,2\}} & 0 & \lambda_{\{3\}} & \lambda_{\{1,2,3\}} \\
0 & 0 & 0 & . & 0 & \lambda_{\{1\}} & \lambda_{\{2\}} & \lambda_{\{1,2,3\}}+\lambda_{\{1,2\}} \\
0 & 0 & 0 & 0 & . & 0 & 0 & \lambda_{\{3\}}+\lambda_{\{1,2,3\}} \\
0 & 0 & 0 & 0 & 0 & \cdot & 0 & \lambda_{\{2\}}+\lambda_{\{1,2,3\}}+\lambda_{\{1,2\}} \\
0 & 0 & 0 & 0 & 0 & 0 & . & \lambda_{\{1\}}+\lambda_{\{1,2,3\}}+\lambda_{\{1,2\}} \\
0 & 0 & 0 & 0 & 0 & 0 & 0 & 0
\end{array}\right]
$$

where '.' represents the sum of all other elements in the row multiplied with -1 . Now, consider group $\{1,2,3\}$. Suppose, that at some point of time obligor 2 is defaulted, but obligors 1 and 3 are still alive, so that process $\mathbf{H}$ is in state $(0,1,0)$. In this case the two survivors in the group $\{1,2,3\}$ may default simultaneously with intensity $\lambda_{\{1,2,3\}}$. Of course, here $\lambda_{\{1,2,3\}}$ cannot be interpreted as intensity of all three defaulting simultaneously, as obligor 2 has already defaulted. In fact, the only state of the model in which $\lambda_{\{1,2,3\}}$ can be interpreted as the intensity of all three defaulting, is state $(0,0,0)$. Note that obligor 1 defaults with intensity $\lambda_{\{1\}}+\lambda_{\{1,2,3\}}+\lambda_{\{1,2\}}$ regardless of the state of the pool, as long company 1 is alive. Similarly, obligor 2 will default with intensity $\lambda_{\{2\}}+\lambda_{\{1,2,3\}}+\lambda_{\{1,2\}}$ regardless of the state of the pool, as long company 1 is alive. Also, obligors 1 and 2 will default together with intensity $\lambda_{\{1,2,3\}}+\lambda_{\{1,2\}}$ regardless of the state of the pool, as long as company 1 and 2 still are alive.

\subsection{Markov Copula Properties}

Below, for every obligor $i$, a real-valued marginal factor process $X^{i}$ will be defined as a suitable function of the above multivariate factor process $\mathbf{X}=\left(X^{Y}\right)_{Y \in \mathcal{Y}}$. We shall then state conditions on the intensities under which the marginal pair $\left(X^{i}, H^{i}\right)$ is a Markov process. Markovianity of the model marginals $\left(X^{i}, H^{i}\right)$ is crucial at the stage of calibration of the model, so that these marginals can be calibrated independently.

Observe that in view of (3), the intensity of a jump of $H^{i}$ from $H_{t-}^{i}=0$ to 1 is given by, for $t \in[0, T]$,

$$
\sum_{\{Y \in \mathcal{Y} ; i \in Y\}} \lambda_{Y}\left(t, X_{t}^{Y}\right),
$$

where the sum in this expression is taken over all pre-specified shocks that can affect name $i$. We define the marginal factor $X^{i}$ as a linear functional $\varphi_{i}$ of the multivariate factor process $\mathbf{X}=\left(X^{Y}\right)_{Y \in \mathcal{Y}}$ so that $X_{t}^{i}:=\varphi_{i}\left(\mathbf{X}_{t}\right)$. In general the transition intensity (9) implies nonMarkovianity of the marginal $\left(X^{i}, H^{i}\right)$. Hence, in order to make the process $\left(X^{i}, H^{i}\right)$ to be 
Markov, one needs to impose a more specified parametrization of (9) as well as conditions on the mapping $\varphi_{i}$. To be more specific:

Assumption 2.5 For every obligor $i$, there exists a linear form $\varphi_{i}(\mathbf{x})$ and a real-valued function $\lambda_{i}(t, x)$ such that for every $(t, \mathbf{x})$ with $\mathbf{x}=\left(x_{Y}\right)_{Y \in \mathcal{Y}}$

$$
\sum_{\{Y \in \mathcal{Y} ; i \in Y\}} \lambda_{Y}\left(t, x_{Y}\right)=\lambda_{i}\left(t, \varphi_{i}(\mathbf{x})\right),
$$

where, in addition, $X_{t}^{i}:=\varphi_{i}\left(\mathbf{X}_{t}\right)$ is a Markov-process with respect to the filtration $\mathcal{F}=$ $\mathcal{F}^{(\mathbf{W}, \mathbf{H})}$, with the following generator acting on functions $v_{i}=v_{i}(t, x)$ with $x \in \mathbb{R}$

$$
A_{t}^{i} v_{i}(t, x)=b_{i}(t, x) \partial_{x} v_{i}(t, x)+\frac{1}{2} \sigma_{i}^{2}(t, x) \partial_{x^{2}}^{2} v_{i}(t, x)
$$

for suitable drift and diffusion coefficients $b_{i}(t, x)$ and $\sigma_{i}(t, x)$.

Note that under such a specification of the intensities, dependence between defaults in the model does not only stem from the possibility of common jumps as in Example 2.4 but it can also come from the factor process $\mathbf{X}$ as in Example 2.7 below.

In the above assumption we require that $X_{t}^{i}=\varphi_{i}\left(\mathbf{X}_{t}\right)$ is a Markov process. This assumption is a non-trivial in general, as a process which is a measurable function of a Markov process does not have to be a Markov process itself. We refer to Pitman and Rogers 33. for some discussion of this issue. In our model set-up one, one can show that under appropriate regularity conditions, if for every $(t, \mathbf{x}, x)$ with $\mathbf{x}=\left(x_{Y}\right)_{Y \in \mathcal{Y}}$ and $x=\varphi_{i}(\mathbf{x})$, one has

$$
\begin{aligned}
& \sum_{\{Y \in \mathcal{Y}\}} b_{Y}(t, \mathbf{x}) \partial_{x_{Y}} \varphi_{i}(\mathbf{x})=b_{i}(t, x) \\
& \sum_{\{Y \in \mathcal{Y}\}} \sigma_{Y}^{2}(t, \mathbf{x})\left(\partial_{x_{Y}} \varphi_{i}(\mathbf{x})\right)^{2}=\sigma_{i}^{2}(t, x)
\end{aligned}
$$

then $X_{t}^{i}=\varphi_{i}\left(\mathbf{X}_{t}\right)$ is an $\mathcal{F}$-Markov process with generator $A^{i}$ in (11). The proof follows from Lemma A.2 (up to the reservation which is made right after the lemma regarding technicalities about the domain of the generators) since for every regular test-function $v_{i}=v_{i}(t, x)$, one has with $u(t, \mathbf{x}):=v_{i}\left(t, \varphi^{i}(\mathbf{x})\right)$

$$
\begin{aligned}
& v_{i}\left(t, X_{t}^{i}\right)-\int_{0}^{t}\left(\partial_{s}+A_{s}^{i}\right) v_{i}\left(s, X_{s}^{i}\right) d s \\
& =u\left(t, \mathbf{X}_{t}\right)-\int_{0}^{t}\left(\partial_{s}+A_{s}\right) u\left(s, \mathbf{X}_{s}\right) d s .
\end{aligned}
$$

In the two examples given below, the $\mathcal{F}$-Markov property of $X_{t}^{i}=\varphi_{i}\left(\mathbf{X}_{t}\right)$ also rigorously follows, in case of Example 2.6 where $\varphi_{i}$ is a coordinate projection operator, from the Markov consistency results of [9], or, in case of Example 2.7, from the semimartingale representation of $X^{i}$ provided by the SDE (14). The $\mathcal{F}$-Markov property of $X^{i}$ in Example 2.7 thus follows from the fact that a strong solution to the Markovian SDE (14) driven by the $\mathcal{F}$-Brownian motion $W^{i}$, is an $\mathcal{F}$-Markov process, by application of Theorem 32 page 100 of Protter [32. Example 2.7 is important, as it goes beyond the case of Example 2.6 where the $\lambda_{I}$ are deterministic functions of time, and it provides a fully stochastic specification of the $\lambda_{Y}$ (including the $\lambda_{I}$ ). 
Example 2.6 (Deterministic Group Intensities) For every group $I \in \mathcal{I}$, the intensity $\lambda_{I}\left(t, x_{I}\right)$ does not depend on $x_{I}$.

Letting $\varphi_{i}(\mathbf{x})=x_{\{i\}}$, then 10$)$ and 12 hold with

$$
\begin{aligned}
\lambda_{i}(t, x) & :=\lambda_{\{i\}}(t, x)+\sum_{\{I \in \mathcal{I} ; i \in I\}} \lambda_{I}(t) \\
b_{i}(t, x) & :=b_{\{i\}}(t, x) \\
\sigma_{i}(t, x) & :=\sigma_{\{i\}}(t, x) .
\end{aligned}
$$

So, $X^{i}=X^{\{i\}}$ is $\mathcal{F}$-Markov with drift and diffusion coefficients $b_{i}(t, x)$ and generator $\sigma_{i}(t, x)$ thus specified.

Example 2.7 (Extended CIR Intensities) For every $Y \in \mathcal{Y}$, the pre-specified group intensities are given by $\lambda_{Y}\left(t, X_{t}^{Y}\right)=X_{t}^{Y}$, where the factor $X^{Y}$ is an extended CIR process

$$
d X_{t}^{Y}=a\left(b_{Y}(t)-X_{t}^{Y}\right) d t+c \sqrt{X_{t}^{Y}} d W_{t}^{Y}
$$

for non-negative constants $a, c$ and non-negative functions $b_{Y}(t)$. The SDEs for the factors $X^{Y}$ have thus the same coefficients except for the $b_{Y}(t)$.

$$
\begin{gathered}
\text { Letting } \varphi_{i}(\mathbf{x})=\sum_{\{Y \in \mathcal{Y} ; i \in Y\}} x_{Y}=x_{\{i\}}+\sum_{\{I \in \mathcal{I} ; i \in I\}} x_{I} \text {, and denoting likewise } b_{i}(t)= \\
\sum_{\{Y \in \mathcal{Y} ; i \in Y\}}(t)=b_{\{i\}}(t)+\sum_{\{I \in \mathcal{I} ; i \in I\}} b_{I}(t) \text {, then } 110 \text { and } 12 \text { hold with } \\
\lambda_{i}(t, x):=x \\
b_{i}(t, x):=a\left(b_{i}(t)-x\right) \\
\sigma_{i}(t, x):=c \sqrt{x} .
\end{gathered}
$$

So, $X^{i}=\sum_{\{Y \in \mathcal{Y} ; i \in Y\}} X^{Y}$ is an $\mathcal{F}$-Markov process with drift and diffusion coefficients $b_{i}(t, x)$ and generator $\sigma_{i}(t, x)$ thus specified.

Note that $X^{i}$ satisfies the following extended CIR SDE with parameters $a, b_{i}(t)$ and $c$ as

$$
d X_{t}^{i}=a\left(b_{i}(t)-X_{t}^{i}\right) d t+c \sqrt{X_{t}^{i}} d W_{t}^{i}
$$

for the $\mathcal{F}$-Brownian motion $W^{i}$ such that

$$
\sqrt{X_{t}^{i}} d W_{t}^{i}=\sum_{i \in Y} \sqrt{X_{t}^{Y}} d W_{t}^{Y}, d W_{t}^{i}=\sum_{i \in Y} \frac{\sqrt{X_{t}^{Y}}}{\sqrt{\sum_{i \in Y} X_{t}^{Y}}} d W_{t}^{Y} .
$$

Remark 2.8 Both the time-deterministic group intensities specification of Example 2.6 and the affine intensities specification of Example 2.7 are used for counterparty credit risk applications in [8, 1, 7] (anticipating the theoretical aspects of the model which are dealt with in the present paper). 
The set of obligors alive (resp. in default) at time $t$ is denoted by $J_{t}=\operatorname{supp}^{c}\left(\mathbf{H}_{t}\right)$ (resp. $H_{t}=\operatorname{supp}\left(\mathbf{H}_{t}\right)$ ). For every $Y \in \mathcal{Y}$ and every set of non-negative constants $t_{i}$, we define the quantities $\Lambda_{s, t}^{Y}, \Lambda_{t}^{Y}$ and $\theta_{t}^{Y}$ as

$$
\Lambda_{s, t}^{Y}=\int_{s}^{t} \lambda_{Y}\left(s, X_{s}^{Y}\right) d s, \Lambda_{t}^{Y}=\Lambda_{0, t}^{Y}=\int_{0}^{t} \lambda_{Y}\left(s, X_{s}^{Y}\right) d s \quad \text { and } \quad \theta_{t}^{Y}=\max _{i \in Y \cap J_{t}} t_{i}
$$

where $Y \cap J_{t}$ in $\theta_{t}^{Y}$ is the set of survivors in $Y$ at time $t$ (and we use in $\theta_{t}^{Y}$ our convention that $\max \emptyset=-\infty)$. Let $\tau_{i}$ denote the default time for obligor $i$. Since $H^{i}$ is the default indicator of name $i$, we have

$$
\tau_{i}=\inf \left\{t>0 ; H_{t}^{i}=1\right\}, H_{t}^{i}=\mathbb{1}_{\left\{\tau_{i} \leq t\right\}} .
$$

The following Proposition gathers the Markov properties of the model.

Proposition 2.9 (i) $(\mathbf{X}, \mathbf{H})$ is an $\mathcal{F}$-Markov process with infinitesimal generator given by $\mathcal{A}$.

(ii) For every obligor $i,\left(X^{i}, H^{i}\right)$ is an $\mathcal{F}$-Markov proces ${ }^{2}$ admitting the following generator acting on functions $u_{i}=u_{i}\left(t, x_{i}, k_{i}\right)$ with $\left(x_{i}, k_{i}\right) \in \mathbb{R} \times\{0,1\}$

$$
\begin{gathered}
\mathcal{A}_{t}^{i} u_{i}\left(t, x_{i}, k_{i}\right)=\quad b_{i}\left(t, x_{i}\right) \partial_{x_{i}} u_{i}\left(t, x_{i}, k_{i}\right)+\frac{1}{2} \sigma_{i}^{2}\left(t, x_{i}\right) \partial_{x_{i}^{2}}^{2} u_{i}\left(t, x_{i}, k_{i}\right) \\
+\lambda_{i}\left(t, x_{i}\right)\left(u_{i}\left(t, x_{i}, 1\right)-u_{i}\left(t, x_{i}, k_{i}\right)\right)
\end{gathered}
$$

Moreover, the $\mathcal{F}$-intensity proces $\S^{3}$ of $H^{i}$ is given by $\mathbb{1}_{\left\{\tau_{i}>t\right\}} \lambda_{i}\left(t, X_{t}^{i}\right)$. In other words, the process $M^{i}$ defined by

$$
M_{t}^{i}=\mathbb{1}_{\left\{\tau_{i} \leq t\right\}}-\int_{0}^{t} \mathbb{1}_{\left\{\tau_{i}>s\right\}} \lambda_{i}\left(s, X_{s}^{i}\right) d s,
$$

is an $\mathcal{F}$-martingale 4

(iii) For any fixed non-negative constants $t, t_{1}, \ldots, t_{n}$, one has

$$
\begin{gathered}
\mathbb{P}\left(\tau_{1}>t_{1}, \ldots, \tau_{n}>t_{n} \mid \mathcal{F}_{t}\right)=\mathbb{P}\left(\tau_{1}>t_{1}, \ldots, \tau_{n}>t_{n} \mid \mathbf{H}_{t}, \mathbf{X}_{t}\right) \\
=\mathbb{1}_{\left\{t_{i}<\tau_{i}, i \in H_{t}\right\}} \mathbb{E}\left\{\exp \left(-\sum_{Y \in \mathcal{Y}} \Lambda_{t, \theta_{t}^{Y}}^{Y}\right) \mid \mathbf{X}_{t}\right\}
\end{gathered}
$$

The conditional survival probability function of every obligor $i$ is given by, for every $t_{i} \geq t$,

$$
\begin{aligned}
\mathbb{P}\left(\tau_{i}>t_{i} \mid \mathcal{F}_{t}\right) & =\mathbb{P}\left(\tau_{i}>t_{i} \mid \mathbf{H}_{t}, \mathbf{X}_{t}\right) \\
& =\mathbb{1}_{\left\{\tau_{i}>t\right\}} \mathbb{E}\left\{\exp \left(-\sum_{Y \in \mathcal{Y}, i \in Y} \Lambda_{t, t_{i}}^{Y}\right) \mid \mathbf{X}_{t}\right\} \\
& =\mathbb{1}_{\left\{\tau_{i}>t\right\}} \mathbb{E}\left\{\exp \left(-\int_{t}^{t_{i}} \lambda_{i}\left(s, X_{s}^{i}\right) d s\right) \mid X_{t}^{i}\right\} \\
& =\mathbb{1}_{\left\{\tau_{i}>t\right\}} G_{t}^{i}\left(t_{i}\right),
\end{aligned}
$$

${ }^{2}$ And hence an $\mathcal{F}^{\left(X^{i}, H^{i}\right)}$-Markov process.

${ }^{3}$ And hence, $\mathcal{F}^{\left(X^{i}, H^{i}\right)}$-intensity process.

${ }^{4}$ And hence, an $\mathcal{F}^{\left(X^{i}, H^{i}\right)}$-martingale. 
with

$$
G_{t}^{i}\left(t_{i}\right)=\mathbb{E}\left\{\exp \left(-\int_{t}^{t_{i}} \lambda_{i}\left(s, X_{s}^{i}\right) d s\right) \mid X_{t}^{i}\right\}
$$

Proof. See Appendix A.2.1.

We shall illustrate part (iii) of the above proposition using the following example.

Example 2.10 In case of two obligors and $\mathcal{Y}=\{\{1\},\{2\},\{1,2\}\}$, one can easily check that (17) boils down to

$$
\begin{gathered}
\mathbb{P}\left(\tau_{1}>t_{1}, \tau_{2}>t_{2} \mid \mathcal{F}_{t}\right)=\mathbb{1}_{\left\{\tau_{1}>t\right\}} \mathbb{1}_{\left\{\tau_{2}>t\right\}} \mathbb{E}\left\{\exp \left(-\sum_{Y \in \mathcal{Y}} \int_{t}^{t_{1} \vee t_{2}} \lambda_{Y}\left(s, X_{s}^{Y}\right)\right) \mid \mathbf{X}_{t}\right\} \\
+\mathbb{1}_{\left\{t_{2}<\tau_{2} \leq t\right\}} \mathbb{1}_{\left\{\tau_{1}>t\right\}} \mathbb{E}\left\{\exp \left(-\int_{t}^{t_{1}} \lambda_{1}\left(s, X_{s}^{1}\right) d s\right) \mid X_{t}^{1}\right\} \\
+\mathbb{1}_{\left\{t_{1}<\tau_{1} \leq t\right\}} \mathbb{1}_{\left\{\tau_{2}>t\right\}} \mathbb{E}\left\{\exp \left(-\int_{t}^{t_{2}} \lambda_{2}\left(s, X_{s}^{2}\right) d s\right) \mid X_{t}^{2}\right\} \\
+\mathbb{1}_{\left\{t_{1}<\tau_{1} \leq t\right\}} \mathbb{1}_{\left\{t_{2}<\tau_{2} \leq t\right\}} .
\end{gathered}
$$

\section{Pricing and Hedging Issues}

This section treats the pricing, calibration and hedging issues in the Markov copula model of Section 2, First, in Subsection 3.1 we derive the price dynamics for CDS contracts and for CDO tranches in this model. In Subsection 3.2 we use dynamics of Subsection 3.1 to derive min-variance hedging strategies in the Markov copula model.

For notational convenience, we assume zero interest rates. The extension of all theoretical results to time dependent, deterministic interest rates is straightforward but more cumbersome notationally, especially regarding hedging. Time-dependent deterministic interest rates will be used in the numerical part.

\subsection{Pricing Equations}

In this subsection we derive price dynamics formulas for CDS contracts and CDO tranches in the Markov model; all prices are considered from perspective of the protection buyers. These dynamics will be useful when deriving the min-variance hedging strategies in Subsection 3.2.

In a zero interest-rates environment, the (ex-dividend) price process of an asset is simply given by the risk neutral conditional expectation of future cash flows associated with the asset; the cumulative value process is the sum of the price process and of the cumulative cash-flows process. The cumulative value process is a martingale, as opposed to the price process. When it comes to hedging, the cumulative value process is the main quantity of interest.

For a fixed maturity $T$, we let $S_{i}$ denote the $T$-year CDS spread for obligor $i$, with recovery rate $R_{i}$. Similarly, we let $S$ denote the $T$-year model CDO tranche spread for the tranche $[a, b]$, with payoff process

$$
L_{t}^{a, b}=L_{a, b}\left(\mathbf{H}_{t}\right)=\left(L_{t}-a\right)^{+}-\left(L_{t}-b\right)^{+},
$$


where $L_{t}=\frac{1}{n} \sum_{i=1}^{n}\left(1-R_{i}\right) H_{t}^{i}$ is the credit loss process for the underlying portfolio. The premium legs in these products are payed at $t_{1}<t_{2}<\ldots<t_{p}=T$ where $t_{j}-t_{j-1}=\mathrm{h}$ and $\mathrm{h}$ is typically a quarter. Below, the notation is the same as in the Itô formula (6).

Proposition 3.1 (i) The price $P^{i}$ and the cumulative value $\widehat{P}^{i}$ at time $t \in[0, T]$ of the single-name $C D S$ on obligor $i$ with contractual spread $S_{i}$ are given by

$$
\begin{aligned}
& P_{t}^{i}=v_{i}\left(t, \mathbf{X}_{t}, \mathbf{H}_{t}\right) \\
& d \widehat{P}_{t}^{i}=\nabla v_{i}\left(t, \mathbf{X}_{t}, \mathbf{H}_{t}\right) \sigma\left(t, \mathbf{X}_{t}\right) d \mathbf{W}_{t}+\sum_{Z \in \mathcal{Z}_{t}} \mathbf{1}_{i \in Z}\left(1-R_{i}-v_{i}\left(t, \mathbf{X}_{t}, \mathbf{H}_{t}\right)\right) d M_{t}^{Z}
\end{aligned}
$$

for a pre-default pricing function $v_{i}(t, \mathbf{x}, \mathbf{k})$ such that

$$
v_{i}\left(t, \mathbf{X}_{t}, \mathbf{H}_{t}\right)=\mathbb{E}\left[-S_{i} \mathrm{~h} \sum_{t<t_{j} \leq T} \mathbb{1}_{\left\{\tau_{i}>t_{j}\right\}}+\left(1-R_{i}\right) \mathbb{1}_{\left\{t<\tau_{i} \leq T\right\}} \mid \mathcal{F}_{t}\right] .
$$

(ii) The price process $\Pi$ and cumulative value $\widehat{\Pi}$ at time $t \in[0, T]$ of a CDO tranche $[a, b]$ with contractual spread $S$ are given by

$$
\begin{aligned}
& \Pi_{t}=u\left(t, \mathbf{X}_{t}, \mathbf{H}_{t}\right) \\
& \begin{aligned}
d \widehat{\Pi}_{t}=\nabla u\left(t, \mathbf{X}_{t}, \mathbf{H}_{t}\right) \sigma\left(t, \mathbf{X}_{t}\right) d \mathbf{W}_{t} \\
\quad+\sum_{Z \in \mathcal{Z}_{t}}\left(L_{a, b}\left(\mathbf{H}_{t-}^{Z}\right)-L_{a, b}\left(\mathbf{H}_{t-}\right)+\delta u^{Z}\left(t, \mathbf{X}_{t}, \mathbf{H}_{t-}\right)\right) d M_{t}^{Z}
\end{aligned}
\end{aligned}
$$

for a pricing function $u(t, \mathbf{x}, \mathbf{k})$ such that

$$
u\left(t, \mathbf{X}_{t}, \mathbf{H}_{t}\right)=\mathbb{E}\left[-S \mathrm{~h} \sum_{t<t_{j} \leq T}\left(b-a-L_{t_{j}}^{a, b}\right)+L_{T}^{a, b}-L_{t}^{a, b} \mid \mathcal{F}_{t}\right] .
$$

Proof. See Appendix A.2.2.

Regarding part (i), note that in view of the marginal Markov properties of the model:

- the pricing function $v_{i}(t, \mathbf{x}, \mathbf{k})$ can essentially be reduced to a "univariate" pre-default pricing function $\tilde{v}_{i}\left(t, x_{i}\right)$;

- the compensated jump martingale in $\widehat{P}^{i}$ can be reduced to a "univariate" martingale representation based on the compensated martingale $M^{i}$ of $H^{i}$ in (16).

However, as will be clear from Subsection 3.2 , the "multivariate" representations of part (i) are more useful in order to handle the hedging issue.

The pricing functions $v_{i}$ and $u$ can be characterized as the unique solutions to the related Kolmogorov equation (42) in Appendix A.2.2 (or, in the CDS case, a "univariate" Kolmogorov equation can be derived to characterize $\tilde{v}_{i}$ ). If the pricing functions are known, the prices at a given time are recovered by plugging the corresponding state of the model into the right-hand-side of the first lines of (21) or (22). But the pricing equation $(42)$ for a CDO tranche leads to a huge system of PDEs which in practice is impossible to handle numerically as soon as $n$ is larger than a few units. As a remedy for this, in Subsection 2.1 of the companion paper [5], we will instead use the translation to a Marshall-Olkin framework which allows us to derive practical recursive pricing schemes for CDO tranche price processes (whereas in practice CDS computations will be based on exponential-affine methodologies). 


\subsection{Min-Variance Hedging}

In this subsection we use the price dynamics from Subsection 3.1 to derive min-variance hedging strategies in the Markov copula model. By min-variance hedging strategies we mean strategies that minimize the variance of the hedging error. Note that in principle one would prefer to minimize the variance relatively to the historical probability measure, however in this paper we minimize the risk-neutral variance for simplicity: see Schweizer 34] for a survey about various quadratic hedging approaches. The hedging strategies are theoretically sound due to our bottom-up Markovian framework and they will be shown in the companion paper [5] to be computationally tractable thanks to the Marshall-Olkin copula interpretation of the model.

Consider a CDO tranche $[a, b]$ with pricing function $u$ specified in Proposition 3.1. Our aim is to find explicit min-variance hedging formulas when hedging this CDO tranche by using the savings account and $d$ single-name CDSs with pricing functions $v_{i}$ given by Proposition 3.1. First we introduce the CDS cumulative value vector-function

$$
\left.\mathbf{v}(t, \mathbf{x}, \mathbf{k})=\left(\mathbb{1}_{k_{1}=0} v_{1}(t, \mathbf{x}, \mathbf{k})+\mathbb{1}_{k_{1}=1}\left(1-R_{1}\right), \ldots, \mathbb{1}_{k_{d}=0} v_{d} t, \mathbf{x}, \mathbf{k}\right)+\mathbb{1}_{k_{d}=1}\left(1-R_{d}\right)\right)^{\top} .
$$

Let $\nabla \mathbf{v}$ denote the Jacobian matrix of $\mathbf{v}$ with respect to $\mathbf{x}$ in the sense of the $d \times \nu$-matrix such that $\nabla \mathbf{v}(t, \mathbf{x}, \mathbf{k})_{i}^{Y}=\partial_{x_{Y}} v_{i}(t, \mathbf{x}, \mathbf{k})$, for every $1 \leq i \leq d$ and $Y \in \mathcal{Y}$. Let $\Delta \mathbf{v}^{Z}$ represent the vector-function of the sensitivities of $\mathbf{v}$ with respect to the event $Z \in \mathcal{N}_{n}$, so

$$
\Delta \mathbf{v}^{Z}(t, \mathbf{x}, \mathbf{k})=\left(\mathbb{1}_{1 \in Z, k_{1}=0}\left(\left(1-R_{1}\right)-v_{1}(t, \mathbf{x}, \mathbf{k})\right), \ldots, \mathbb{1}_{d \in Z, k_{d}=0}\left(\left(1-R_{d}\right)-v_{d}(t, \mathbf{x}, \mathbf{k})\right)\right)^{\top} .
$$

By using the vector notation $\widehat{\mathbf{P}}=\left(\widehat{P}^{i}\right)_{1 \leq i \leq d}$, one has in view of Proposition 3.1(i)

$$
d \widehat{\mathbf{P}}_{t}=\nabla \mathbf{v}\left(t, \mathbf{X}_{t}, \mathbf{H}_{t}\right) \sigma\left(t, \mathbf{X}_{t}\right) d \mathbf{W}_{t}+\sum_{Z \in \mathcal{Z}_{t}} \Delta \mathbf{v}^{Z}\left(t, \mathbf{X}_{t}, \mathbf{H}_{t-}\right) d M_{t}^{Z}
$$

Let

$$
\Delta u^{Z}(t, \mathbf{x}, \mathbf{k})=\delta^{Z} u(t, \mathbf{x}, \mathbf{k})+L_{a, b}\left(\mathbf{k}^{Z}\right)-L_{a, b}(\mathbf{k})
$$

represent the function of sensitivity of the CDO tranche $[a, b]$ cumulative value process with respect to the event $Z \in \mathcal{N}_{n}$. Let $\zeta$ be an $d$-dimensional row-vector process, representing the number of units held in the first $d$ CDSs which are used in a self-financing 5 hedging strategy for the CDO tranche $[a, b]$. Given $(22)$ and $(23)$, the tracking error $\left(e_{t}\right)$ of the hedged portfolio satisfies $e_{0}=0$ and, for $t \in[0, T]$

$$
\begin{aligned}
d e_{t}= & d \widehat{\Pi}_{t}-\zeta_{t} d \widehat{\mathbf{P}}_{t} \\
=( & \left.\nabla u\left(t, \mathbf{X}_{t}, \mathbf{H}_{t}\right)-\zeta_{t} \nabla \mathbf{v}\left(t, \mathbf{X}_{t}, \mathbf{H}_{t}\right)\right) \sigma\left(t, \mathbf{X}_{t}\right) d \mathbf{W}_{t} \\
& \quad+\sum_{Z \in \mathcal{Z}_{t}}\left(\Delta u^{Z}\left(t, \mathbf{X}_{t}, \mathbf{H}_{t-}\right)-\zeta_{t} \Delta \mathbf{v}^{Z}\left(t, \mathbf{X}_{t}, \mathbf{H}_{t-}\right)\right) d M_{t}^{Z}
\end{aligned}
$$

Since the martingale dimension of the model is $\nu+2^{n}$, replication is typically out-of-reach 6 in the Markov model. However, in view of (24), we can find min-variance hedging formulas.

\footnotetext{
${ }^{5}$ Using also the savings account (constant asset)

${ }^{6}$ See the comments following Proposition 2.3
} 
Proposition 3.2 The min-variance hedging strategy $\zeta$ is

$$
\zeta_{t}=\frac{d\langle\widehat{\Pi}, \widehat{\mathbf{P}}\rangle_{t}}{d t}\left(\frac{d\langle\widehat{\mathbf{P}}\rangle_{t}}{d t}\right)^{-1}=\zeta\left(t, \mathbf{X}_{t}, \mathbf{H}_{t-}\right)
$$

where $\zeta=(u, \mathbf{v})(\mathbf{v}, \mathbf{v})^{-1}$, with

$$
\begin{aligned}
& (u, \mathbf{v})=(\nabla u) \sigma^{2}(\nabla \mathbf{v})^{\top}+\sum_{Y \in \mathcal{Y}} \lambda_{Y} \Delta u^{Y}\left(\Delta \mathbf{v}^{Y}\right)^{\top} \\
& (\mathbf{v}, \mathbf{v})=(\nabla \mathbf{v}) \sigma^{2}(\nabla \mathbf{v})^{\top}+\sum_{Y \in \mathcal{Y}} \lambda_{Y} \Delta \mathbf{v}^{Y}\left(\Delta \mathbf{v}^{Y}\right)^{\top} .
\end{aligned}
$$

Proof. The first identity in (25) is a classical risk neutral min-variance hedging 7 formula, derived for instance in Section 4.2.3.1 of Crépey [18. Moreover, one has by computation of the oblique brackets based on the second lines in (21) and (22):

$$
\begin{aligned}
& \frac{d\langle\widehat{\Pi}, \widehat{\mathbf{P}}\rangle_{t}}{d t}=\left((\nabla u) \sigma^{2}(\nabla \mathbf{v})^{\top}+\sum_{Z \in \mathcal{Z}_{t}} \lambda_{Z} \Delta u^{Z}\left(\Delta \mathbf{v}^{Z}\right)^{\top}\right)\left(t, \mathbf{X}_{t}, \mathbf{H}_{t-}\right)=(u, \mathbf{v})\left(t, \mathbf{X}_{t}, \mathbf{H}_{t-}\right) \\
& \frac{d\langle\widehat{\mathbf{P}}\rangle_{t}}{d t}=\left((\nabla \mathbf{v}) \sigma^{2}(\nabla \mathbf{v})^{\top}+\sum_{Z \in \mathcal{Z}_{t}} \lambda_{Z} \Delta \mathbf{v}^{Z}\left(\Delta \mathbf{v}^{Z}\right)^{\top}\right)\left(t, \mathbf{X}_{t}, \mathbf{H}_{t-}\right)=(\mathbf{v}, \mathbf{v})\left(t, \mathbf{X}_{t}, \mathbf{H}_{t-}\right)
\end{aligned}
$$

where the second identities in both lines of $(27)$ use simplifications similar to those used in the proof of the Itô formula (6) in Appendix A.1.2.

In (26), the $u$-related terms can be computed by using the conditional convolutionrecursion procedures developed in the companion paper [5]; the $v_{i}$-related terms can be computed very quickly (actually semi-explicitly in either of the specifications of examples 2.6 and 2.7). We will illustrate in [5] the tractability of this approach for computing minvariance hedging deltas.

We refer the reader to Elouerkhaoui [21] for analogous formulas. A nice feature of our set-up however is that due to the specific structure of the intensities, the sums in (26) are over the set $\mathcal{Y}$ of shocks $\mathcal{Y}$ which is of cardinality $\nu=n+m$, as opposed to the set $\mathcal{N}_{n}$ of all set-events $Z$ in $[21$.

We also refer the reader to Frey and Backhaus [23] for other related min-variance hedging formulas.

\section{A Appendix}

\section{A.1 Model Construction}

The point process $\mathbf{H}$ with intensity depending on the factor process $\mathbf{X}$ in (3), is constructed by an $\mathbf{X}$-related change of probability measure, starting from an independent continuoustime Markov chain under an auxiliary probability measure $\widehat{\mathbb{P}}$. So, given a factor process $\mathbf{X}$ as in (1) where $\mathbf{W}$ is a $\widehat{\mathbb{P}}$-Brownian motion, let $\mathbf{H}$ denote a continuous-time Markov chain with $\widehat{\mathbb{P}}$-intensity one of transition from $\mathbf{k}$ to $\mathbf{l}$, for every $\mathbf{l} \neq \mathbf{k}$. Let then the $\widehat{\mathbb{P}}$-martingale

\footnotetext{
${ }^{7}$ See Schweizer 34

${ }^{8}$ Under suitable regularity and growth assumptions on the model coefficients, see Ethier and Kurtz 22 or Crépey [18.
} 
$\Gamma$ be defined by $\Gamma_{0}=1$ and, for $t \in[0, T]$,

$$
\begin{aligned}
\frac{d \Gamma_{t}}{\Gamma_{t-}} & =\sum_{l \in\{0,1\}^{n}}\left(\lambda\left(t, \mathbf{X}_{t}, \mathbf{H}_{t-}, \mathbf{l}\right)-1\right)\left(d N_{t}\left(\mathbf{H}_{t-}, \mathbf{l}\right)-\mathbb{1}_{\mathbf{l} \neq \mathbf{H}_{t-}} d t\right) \\
& =\sum_{\mathbf{l} \neq \mathbf{H}_{t-}}\left(\lambda\left(t, \mathbf{X}_{t}, \mathbf{H}_{t-}, \mathbf{l}\right)-1\right)\left(d N_{t}\left(\mathbf{H}_{t-}, \mathbf{l}\right)-d t\right),
\end{aligned}
$$

where the functions $\lambda(t, \mathbf{x}, \mathbf{k}, \mathbf{l})$ are those of $(3)$, and where $N_{t}(\mathbf{k}, \mathbf{l})$ is the point process with $\widehat{\mathbb{P}}$-intensity $\mathbb{1}_{\left\{\mathbf{k}=\mathbf{H}_{t-}, \mathbf{l} \neq \mathbf{k}\right\}}$ counting the transitions of $\mathbf{H}$ from $\mathbf{k}$ to $\mathbf{l}$, for every $\mathbf{k}, \mathbf{l} \in\{0,1\}^{n}$. Defining the measure $\mathbb{P}$ by $\frac{d \mathbb{P}}{d \widehat{\mathbb{P}}}=\Gamma_{T}$, it is then standard to check $\mathrm{l}^{9}$ that the point process $\mathbf{H}$ has intensity (3) under $\mathbb{P}$. To be precise the intensity of $N_{t}(\mathbf{k}, \mathbf{l})$ is given by (3), with respect to the model filtration $\mathcal{F}=\mathcal{F}^{(\mathbf{W}, \mathbf{H})}$, and the probability measure $\mathbb{P}$. Moreover, process $\mathbf{W}$ remains a Brownian motion under $\mathbb{P}$, the measure-change preserves Markov property of $\mathbf{X}$ with respect to filtration $\mathcal{F}$, and the generator of $\mathbf{X}$ under the new measure is still $A_{t}$.

Note that since martingale representation holds under $\widehat{\mathbb{P}} \cdot 10$ martingale representation also holds under the equivalent measure $\mathbb{P}$.

Remark A.1 The prevailing risk neutral probability measure in the paper is $\mathbb{P}$, whereas the auxiliary measure $\widehat{\mathbb{P}}$ is only a mathematical tool used for constructing the model, with no particular financial interpretation.

\section{A.1.1 Proof of Lemma 2.2}

By definition of the set-event indicator process $H^{Z}$, where $Z \in \mathcal{N}_{n}$, one has in our model, for $t \in[0, T]$,

$$
d H_{t}^{Z}=\sum_{\left\{\mathbf{k}, \mathbf{l} \in\{0,1\}^{n} ; \operatorname{supp}(\mathbf{l}) \backslash \operatorname{supp}(\mathbf{k})=Z\right\}} d N_{t}(\mathbf{k}, \mathbf{l}) .
$$

So, by (3),

$$
\begin{aligned}
\ell_{t}^{Z} & =\sum_{\left\{\mathbf{k}, \mathbf{l} \in\{0,1\}^{n} ; \operatorname{supp}(\mathbf{l}) \backslash \operatorname{supp}(\mathbf{k})=Z\right\}} \sum_{\left\{\mathbf{l} \in\{0,1\}^{n} ; \operatorname{supp}(\mathbf{l}) \backslash \operatorname{supp}\left(\mathbf{H}_{t-}\right)=Z\right\}} \sum_{\left\{Y \in \mathcal{Y} ; \mathbf{H}_{t-}^{Y}=\mathbf{l}\right\}} \lambda_{\left\{Y \in \mathcal{Y} ; \mathbf{k}^{Y}=\mathbf{l}\right\}} \lambda_{Y}\left(t, X_{t}^{Y}\right) \\
= & \sum_{\left\{Y \in \mathcal{Y} ; \operatorname{supp}\left(\mathbf{H}_{t-}^{Y}\right) \backslash \operatorname{supp}\left(\mathbf{H}_{t-}\right)=Z\right\}} \lambda_{Y}\left(t, X_{t}^{Y}\right) \\
= & \sum_{\left\{Y \in \mathcal{Y} ; Y_{t}=Z\right\}} \lambda_{Y}\left(t, X_{t}^{Y}\right) .
\end{aligned}
$$

\footnotetext{
${ }^{9}$ See for instance the proof of Lemma 12.3.5 in Crépey [18].

${ }^{10}$ In virtue of standard arguments, see for instance Chapter 10 of [27].
} 


\section{A.1.2 Proof of Proposition 2.3}

Observe that $\left[M^{Y}, M^{Z}\right]=0$ for $Y \neq Z$. One thus has the following Itô formula (see for instance Theorem 3.89 page 109 of Jacod [26] or Crépey [18])

$$
\begin{aligned}
& d u\left(t, \mathbf{X}_{t}, \mathbf{H}_{t}\right)=\left(\partial_{t}+A_{t}\right) u\left(t, \mathbf{X}_{t}, \mathbf{H}_{t}\right) d t+\nabla u\left(t, \mathbf{X}_{t}, \mathbf{H}_{t}\right) \sigma\left(t, \mathbf{X}_{t}\right) d \mathbf{W}_{t} \\
& +\sum_{Z \in \mathcal{N}_{n}} \delta u^{Z}\left(t, \mathbf{X}_{t}, \mathbf{H}_{t-}\right) d H_{t}^{Z}
\end{aligned}
$$

where we write

$$
A_{t} u(t, \mathbf{x}, \mathbf{k})=\sum_{Y \in \mathcal{Y}}\left(b_{Y}\left(t, x_{Y}\right) \partial_{x_{Y}} u(t, \mathbf{x}, \mathbf{k})+\frac{1}{2} \sigma_{Y}^{2}\left(t, x_{Y}\right) \partial_{x_{Y}^{2}}^{2} u(t, \mathbf{x}, \mathbf{k})\right) .
$$

Moreover, the structure (4) of the set intensities implies that

$$
\sum_{Z \in \mathcal{N}_{n}} \delta u^{Z}\left(t, \mathbf{X}_{t}, \mathbf{H}_{t-}\right) d H_{t}^{Z}=\sum_{Z \in \mathcal{Z}_{t}} \delta u^{Z}\left(t, \mathbf{X}_{t}, \mathbf{H}_{t-}\right) d H_{t}^{Z}
$$

which we may further rewrite as

$$
\begin{aligned}
& \sum_{Z \in \mathcal{Z}_{t}} \ell_{Z}\left(t, \mathbf{X}_{t}, \mathbf{H}_{t-}\right) \delta u^{Z}\left(t, \mathbf{X}_{t}, \mathbf{H}_{t-}\right) d t \\
& \quad+\sum_{Z \in \mathcal{Z}_{t}}\left(\delta u^{Z}\left(t, \mathbf{X}_{t}, \mathbf{H}_{t-}\right) d H_{t}^{Z}-\ell_{Z}\left(t, \mathbf{X}_{t}, \mathbf{H}_{t}\right) \delta u^{Z}\left(t, \mathbf{X}_{t}, \mathbf{H}_{t}\right) d t\right)
\end{aligned}
$$

Here the second term is $\sum_{Z \in \mathcal{Z}_{t}} \delta u^{Z}\left(t, \mathbf{X}_{t}, \mathbf{H}_{t-}\right) d M_{t}^{Z}$, whereas one has by (4) in the first term:

$$
\begin{aligned}
\sum_{Z \in \mathcal{Z}_{t}} & \ell_{Z}\left(t, \mathbf{X}_{t}, \mathbf{H}_{t-}\right) \delta u^{Z}\left(t, \mathbf{X}_{t}, \mathbf{H}_{t-}\right) \\
= & \sum_{Z \in \mathcal{Z}_{t}} \sum_{Y \in \mathcal{Y} ; Y_{t}=Z} \lambda_{Y}\left(t, X_{t}^{Y}\right) \delta u^{Z}\left(t, \mathbf{X}_{t}, \mathbf{H}_{t-}\right) \\
= & \sum_{Y \in \mathcal{Y}} \lambda_{Y}\left(t, X_{t}^{Y}\right) \delta u^{Y}\left(t, \mathbf{X}_{t}, \mathbf{H}_{t-}\right)
\end{aligned}
$$

using in the last identity that

$$
\delta u^{Z}(t, \mathbf{x}, \mathbf{k})=\delta u^{Y}(t, \mathbf{x}, \mathbf{k}),
$$

for every $t, \mathbf{x}, \mathbf{k}, Y$ and $Z$ such that $Y_{t}=Z$. Thus (28) indeed reduces to (6).

\section{A.2 Markov Properties}

Let us first recall the following local martingale characterization of a Markov process with generator $\mathcal{L}$. We work under the standing assumption that uniqueness holds for the solution of the martingale problem defined by $\mathcal{L}$.

Lemma A.2 (See, e.g., Ethier and Kurtz [22]) Let $X$ be a right-continuous process with Euclidean state space $E$, adapted to some filtration $\mathcal{F}$. For $X$ to be an $\mathcal{F}$-Markov 
process with infinitesimal generator $\mathcal{L}$, it is necessary and sufficient that, for every realvalued function $\varphi$ in the domain of $\mathcal{L}$,

$$
\varphi\left(t, X_{t}\right)-\int_{0}^{t}\left(\partial_{s}+\mathcal{L}_{s}\right) \varphi\left(s, X_{s}\right) d s
$$

is an $\mathcal{F}$ - local martingale.

We shall use this characterization informally in this paper, ignoring the technicalities related to the notion of domain of an operator. Furthermore, throughout the paper we work under the standing assumption that the valuation equation associated to any infinitesimal generator that we use, is well posed in an appropriate functional space. Finally, we assume that uniqueness holds for the solution of the related martingale problem. The reader is referred to Ethier and Kurtz [22] for more details and for specific conditions which can be postulated in these regards.

\section{A.2.1 Proof of Proposition 2.9}

(i) In view of the Itô formula $(6),(\mathbf{X}, \mathbf{H})$ solves the martingale problem with generator $\mathcal{A}$ in the filtration $\mathcal{F}$, and is thus an $\mathcal{F}$-Markov process.

(ii) By application of the local martingale characterization of an $\mathcal{F}$-Markov process $(\mathbf{X}, \mathbf{H})$ with generator $\mathcal{A}$ to test-functions of the form $u(t, \mathbf{x}, \mathbf{k})=v_{i}\left(t, x_{i}, k_{i}\right)$, we get the local martingale characterization of an $\mathcal{F}$ - Markov process with generator $\mathcal{A}^{i}$ for $\left(X^{i}, H^{i}\right)$. Considering $v_{i}\left(t, x_{i}, k_{i}\right)=\mathbb{1}_{k_{i}=1}$ therein yields that $M^{i}$ in $(16)$ is an $\mathcal{F}$-local martingale.

(iii) We denote $t_{Z}=\max _{i \in Z} t_{i}$, for every $Z \in \mathcal{N}_{n}$. Formula (17) follows directly from Lemma A.3 below since one has, for every $t, t_{1}, \ldots, t_{n} \geq 0$,

$$
\begin{aligned}
\mathbb{P}\left(\tau_{1}>t_{1}, \ldots, \tau_{n}>t_{n} \mid \mathcal{F}_{t}\right) & =\sum_{Z \in \mathcal{N}_{n}} \mathbb{1}_{\left\{J_{t}=Z\right\}} \mathbb{P}\left(\tau_{1}>t_{1}, \ldots, \tau_{n}>t_{n} \mid \mathcal{F}_{t}\right) \\
& =\sum_{Z \in \mathcal{N}_{n}}\left(\prod_{i \notin Z} \mathbb{1}_{t_{i}<\tau_{i} \leq t}\right) \mathbb{E}\left\{\prod_{i \in Z} \mathbb{1}_{\tau_{i}>t_{i} \vee t} \mid \mathcal{F}_{t}\right\}
\end{aligned}
$$

and

$$
\begin{aligned}
\mathbb{1}_{\left\{t_{i}<\tau_{i}, i \in H_{t}\right\}} \mathbb{E}\left\{\exp \left(-\sum_{Y \in \mathcal{Y}} \Lambda_{t, \theta_{t}^{Y}}^{Y}\right) \mid \mathbf{X}_{t}\right\} \\
=\sum_{Z \in \mathcal{N}_{n}} \mathbb{1}_{\left\{J_{t}=Z\right\}} \mathbb{1}_{\left\{t_{i}<\tau_{i}, i \notin Z\right\}} \mathbb{E}\left\{\exp \left(-\sum_{Y \in \mathcal{Y}} \Lambda_{t, \theta_{t}^{Y}}^{Y}\right) \mid \mathbf{X}_{t}\right\} \\
=\sum_{Z \in \mathcal{N}_{n}}\left(\prod_{i \notin Z} \mathbb{1}_{t_{i}<\tau_{i} \leq t}\right)\left(\prod_{i \in Z} \mathbb{1}_{\tau_{i}>t}\right) \mathbb{E}\left\{\exp \left(-\sum_{Y \in \mathcal{Y}} \Lambda_{t, t_{Y \cap Z}}^{Y}\right) \mid \mathbf{X}_{t}\right\} .
\end{aligned}
$$

Given (17), the other formulas of part (iii) in Proposition 2.9 are straightforward.

Lemma A.3 For every $t, t_{1}, \ldots, t_{n} \geq 0$, and for every $Z \in \mathcal{N}_{n}$, one has,

$$
\mathbb{E}\left\{\prod_{i \in Z} \mathbb{1}_{\tau_{i}>t_{i} \vee t} \mid \mathcal{F}_{t}\right\}=\left(\prod_{i \in Z} \mathbb{1}_{\tau_{i}>t}\right) \mathbb{E}\left\{\exp \left(-\sum_{Y \in \mathcal{Y} ; Y \cap Z \neq \emptyset} \Lambda_{t, t_{Y}}^{Y}\right) \mid \mathbf{X}_{t}\right\}
$$


Proof. It is enough to prove that for $t_{i} \geq t$ one has, for every $Z \in \mathcal{N}_{n}$,

$$
\mathbb{E}\left\{\prod_{i \in Z} \mathbb{1}_{\tau_{i}>t_{i}} \mid \mathcal{F}_{t}\right\}=\left(\prod_{i \in Z} \mathbb{1}_{\tau_{i}>t}\right) \mathbb{E}\left\{\exp \left(-\sum_{Y \in \mathcal{Y}} \Lambda_{t, t_{Y \cap Z}}^{Y}\right) \mid \mathbf{X}_{t}\right\} .
$$

Indeed, for general $t_{i}$, applying $(32)$ to the $t_{i} \vee t$ yields

$$
\begin{gathered}
\mathbb{E}\left\{\prod_{i \in Z} \mathbb{1}_{\tau_{i}>t_{i} \vee t} \mid \mathcal{F}_{t}\right\}=\left(\prod_{i \in Z} \mathbb{1}_{\tau_{i}>t}\right) \mathbb{E}\left\{\exp \left(-\sum_{Y \in \mathcal{Y}} \Lambda_{t, \max _{i \in Y \cap Z} t_{i} \vee t}^{Y}\right) \mid \mathbf{X}_{t}\right\} \\
=\left(\prod_{i \in Z} \mathbb{1}_{\tau_{i}>t}\right) \mathbb{E}\left\{\exp \left(-\sum_{Y \in \mathcal{Y} ; Y \cap Z \neq \emptyset} \Lambda_{t, \max _{i \in Z} t_{i}}^{Y}\right) \mid \mathbf{X}_{t}\right\},
\end{gathered}
$$

which is (31). Let us thus show (32) for $t_{i} \geq t$, by induction on the cardinality $d$ of $Z$. For $d=0$, the result is trivial. Assuming the result at rank $d-1 \geq 0$, let us show the result at rank $d$. Let us suppose, without loss of generality, that $Z=N_{d}$ and $t_{1} \geq t_{2} \geq \cdots \geq t_{d} \geq t$. One then needs to prove that, using the notation $J^{l}=1-H^{l}$ for every $l \in N_{d}$,

$$
\mathbb{E}\left(\prod_{l=1}^{d} J_{t_{l}}^{l} \mid \mathcal{F}_{t}\right)=\left(\prod_{l=1}^{d} J_{t}^{l}\right) \mathbb{E}\left\{\exp \left(-\sum_{Y \in \mathcal{Y}} \Lambda_{t, t_{N_{d}} \cap Y}^{Y}\right) \mid \mathbf{X}_{t}\right\} .
$$

To establish (33) one first observes that

$$
\mathbb{E}\left(\prod_{l=1}^{d} J_{t_{l}}^{l} \mid \mathcal{F}_{t}\right)=\mathbb{E}\left\{J_{t_{d}}^{d} \mathbb{E}\left\{\prod_{l=1}^{d-1} J_{t_{l}}^{l} \mid \mathcal{F}_{t_{d}}\right\} \mid \mathcal{F}_{t}\right\}
$$

where by the induction hypothesis at rank $d-1$ the inner conditional expectation can be represented as

$$
\left(\prod_{l=1}^{d-1} J_{t_{d}}^{l}\right) \mathbb{E}\left\{\exp \left(-\sum_{Y \in \mathcal{Y}} \Lambda_{t_{d}, t_{N_{d-1} \cap Y}}^{Y}\right) \mid \mathbf{X}_{t_{d}}\right\}=\left(\prod_{l=1}^{d-1} J_{t_{d}}^{l}\right) v\left(t_{d}, \mathbf{X}_{t_{d}}\right)
$$

for a suitable function $v=v(t, \mathbf{x})$ over $\left[0, t_{d-1}\right] \times \mathbb{R}^{\mathcal{Y}}$, by the Markov property of $\mathbf{X}$. Here the upper bound $t_{d-1}$ for the domain of definition of the function $v$ follows from the fact that $t_{d} \leq t_{d-1} \leq t_{N_{d-1} \cap Y}$, for every $Y \in \mathcal{Y}$ with $N_{d-1} \cap Y \neq \emptyset$. Inserting (35) into (34) yields by the Markov property of $(\mathbf{X}, \mathbf{H})$ that

$$
\mathbb{E}\left(\prod_{l=1}^{d} J_{t_{l}}^{l} \mid \mathcal{F}_{t}\right)=\mathbb{E}\left\{\left(\prod_{l=1}^{d} J_{t_{d}}^{l}\right) v\left(t_{d}, \mathbf{X}_{t_{d}}\right) \mid \mathcal{F}_{t}\right\}=u\left(t, \mathbf{X}_{t}, \mathbf{H}_{t}\right),
$$

for a function $u=u(t, \mathbf{x}, \mathbf{k})$ over $\left[0, t_{d}\right] \times \mathbb{R}^{\mathcal{Y}} \times\{0,1\}^{n}$ characterized by:

$$
\left\{\begin{array}{l}
u\left(t_{d}, \mathbf{x}, \mathbf{k}\right)=\left(\prod_{l=1}^{d}\left(1-k_{l}\right)\right) v\left(t_{d}, \mathbf{x}\right), \quad \mathbf{x}=\left(x_{Y}\right)_{Y \in \mathcal{Y}}, \mathbf{k}=\left(k_{1}, \ldots, k_{n}\right) \in\{0,1\}^{n} \\
\left(\partial_{t}+\mathcal{A}_{t}\right) u(t, \mathbf{x}, \mathbf{k})=0, \quad t<t_{d}, \mathbf{x}=\left(x_{Y}\right)_{Y \in \mathcal{Y}}, \mathbf{k} \in\{0,1\}^{n}
\end{array}\right.
$$

One finally shows that the RHS in (33) admits a representation of the form $\left(\prod_{l=1}^{d} J_{t}^{l}\right) w\left(t, \mathbf{X}_{t}\right)$, where the function $\tilde{u}(t, \mathbf{x}, \mathbf{k})=\left(\prod_{l=1}^{d}\left(1-k_{l}\right)\right) w(t, \mathbf{x})$ solves $(36)$. By our standing assumption in this paper equation (36) has a unique solution. Thus $\tilde{u}=u$, which proves (33). 
Since $\mathbf{X}$ is Markov with generator $A$ (cf. (i)), the conditional expectation in the RHS of (33) can be represented as $w\left(t, \mathbf{X}_{t}\right)$, for a deterministic function $w=w(t, \mathbf{x})$ over the domain $\left[0, t_{d}\right] \times \mathbb{R}^{\mathcal{Y}}$. In order to get the analytic characterization of $w$, first note that for every $Y \in \mathcal{Y}$, one has:

$$
\Lambda_{t_{d}, t_{N_{d-1} \cap Y}}^{Y}=\Lambda_{t_{d}, t_{N_{d} \cap Y}}^{Y}=\Lambda_{t_{d}, t_{N_{d} \cap Y}}^{Y} .
$$

This yields the terminal condition $w\left(t_{d}, \mathbf{x}\right)=v\left(t_{d}, \mathbf{x}\right), \mathbf{x}=\left(x_{Y}\right)_{Y \in \mathcal{Y}}$. One further has by an application of the Feynman-Kac formula that (see, e.g., Jeanblanc et al. [27])

$$
\left(\partial_{t}+A_{t}\right) w(t, \mathbf{x})=\left(\sum_{Y \in \mathcal{Y} ; Y \cap Z \neq \emptyset} \lambda_{Y}\left(t, x_{Y}\right)\right) w(t, \mathbf{x}), \quad t<t_{d}, \mathbf{x}=\left(x_{Y}\right)_{Y \in \mathcal{Y}} .
$$

As a result the function $w=w(t, \mathbf{x})$ is the solution on $\left[0, t_{d}\right] \times \mathbb{R}^{\mathcal{Y}}$ to the following Kolmogorov pricing PDE:

$$
\left\{\begin{array}{l}
w\left(t_{d}, \mathbf{x}\right)=v\left(t_{d}, \mathbf{x}\right), \quad \mathbf{x}=\left(x_{Y}\right)_{Y \in \mathcal{Y}} \\
\left(\partial_{t}+A_{t}\right) w(t, \mathbf{x})=\left(\sum_{Y \in \mathcal{Y} ; Y \cap Z \neq \emptyset} \lambda_{Y}\left(t, x_{Y}\right)\right) w(t, \mathbf{x}), \quad t<t_{d}, \mathbf{x}=\left(x_{Y}\right)_{Y \in \mathcal{Y} .}
\end{array}\right.
$$

Denoting $\tilde{u}(t, \mathbf{x}, \mathbf{k})=\left(\prod_{l \in N_{d}}\left(1-k_{l}\right)\right) w(t, \mathbf{x})$, an application of the operator $\mathcal{A}_{t}$ of (7) yields:

$$
\begin{aligned}
\left(\partial_{t}+\mathcal{A}_{t}\right) \tilde{u}(t, \mathbf{x}, \mathbf{k})= & \left(\prod_{l \in N_{d}}\left(1-k_{l}\right)\right)\left(\partial_{t}+A_{t}\right) w(t, \mathbf{x})+w(t, \mathbf{x}) \times \\
& \times \sum_{Y \in \mathcal{Y}} \lambda_{Y}\left(t, x_{Y}\right)\left(\left(\prod_{l \in N_{d}}\left(1-k_{l}^{Y}\right)\right)-\prod_{l \in N_{d}}\left(1-k_{l}\right)\right),
\end{aligned}
$$

where we set, for $Y \in \mathcal{Y}$ and $l \in N_{d}$,

$$
k_{l}^{Y}= \begin{cases}1, & Y \ni l \\ k_{l}, & \text { else }\end{cases}
$$

Therefore

$$
\begin{aligned}
\sum_{Y \in \mathcal{Y}} \lambda_{Y}\left(t, x_{Y}\right)(( & \left.\left.\prod_{l \in N_{d}}\left(1-k_{l}^{Y}\right)\right)-\prod_{l \in N_{d}}\left(1-k_{l}\right)\right) \\
& =-\prod_{l \in N_{d}}\left(1-k_{l}\right) \sum_{Y \in \mathcal{Y} ; Y \cap N_{d} \neq \emptyset} \lambda_{Y}\left(t, x_{Y}\right) .
\end{aligned}
$$

Plugging (37) and (39) in the RHS of $(38)$ yields that $\left(\partial_{t}+\mathcal{A}_{t}\right) \tilde{u}(t, \mathbf{x}, \mathbf{k})=0$. Finally $\tilde{u}$ solves (36), which finishes the demonstration.

\section{A.2.2 Proof of Proposition 3.1}

Given a function $f=f(t, y)$, let $f\left(t_{j}-, x\right)$ be a notation for the formal limit

$$
\lim _{(t, y) \rightarrow\left(t_{j}, x\right) \text { with } t<t_{j}} f(t, y) .
$$

In view of the Markov property of the model, the following lemma holds in virtue of the Feynman-Kac formula.11

\footnotetext{
${ }^{11}$ See, e.g., Jeanblanc et al. [27].
} 
Lemma A.4 Given real-valued functions $\phi(\mathbf{k})$ and $\psi(\mathbf{k})$, one has $\mathbb{E}\left[\sum_{t<t_{j} \leq T} \phi\left(\mathbf{H}_{t_{j}}\right)+\right.$ $\left.\psi\left(\mathbf{H}_{T}\right) \mid \mathcal{F}_{t}\right]=w\left(t, \mathbf{X}_{t}, \mathbf{H}_{t}\right)$, where the function $w(t, \mathbf{x}, \mathbf{k})$ is the solution to the following Kolmogorov pricing PDE system: $w(T, \mathbf{x}, \mathbf{k})=\psi(\mathbf{k}), \mathbf{x}=\left(x_{Y}\right)_{Y \in \mathcal{Y}}, \mathbf{k} \in\{0,1\}^{n}$, and for $j$ decreasing from $p$ to 1 :

- At $t=t_{j}$,

$$
w\left(t_{j}-, \mathbf{x}, \mathbf{k}\right)=w\left(t_{j}, \mathbf{x}, \mathbf{k}\right)+\phi(\mathbf{k}), \mathbf{x}=\left(x_{Y}\right)_{Y \in \mathcal{Y}}, \mathbf{k} \in\{0,1\}^{n},
$$

- On the time interval $\left[t_{j-1}, t_{j}\right)$,

$$
\left(\partial_{t}+\mathcal{A}_{t}\right) w(t, \mathbf{x}, \mathbf{k})=0, \mathbf{x}=\left(x_{Y}\right)_{Y \in \mathcal{Y}}, \mathbf{k} \in\{0,1\}^{n} .
$$

Applying this lemma with

$$
\psi_{i}=\left(1-R_{i}\right), \phi_{i}=-S_{i} \mathrm{~h}
$$

for part (i) and

$$
\psi=L_{a, b}, \quad \phi=-S \mathrm{~h}\left(b-a-L_{a, b}\right)
$$

for part (ii) establishes the first lines in identities (21) and (22). Regarding the latter, note that the ex-dividend pricing function $u(t, \mathbf{k}, \mathbf{x})$ in $(22)$, is provided by $w(t, \mathbf{k}, \mathbf{x})-L_{a, b}(\mathbf{k})$ here.

Moreover, in the filtration $\mathcal{F}=\mathcal{F}^{\mathbf{W}, \mathbf{H}}$, a martingale can only jump at totally unpredictable stopping times. In particular, the cumulative value processes cannot jump at the fixed times $t_{j}$. Given the first lines in (21) and (22), the second lines then readily follow using the Itô formula (6).

\section{References}

[1] Assefa, S., Bielecki, T.R., Crépey, S. and Jeanblanc, M.: CVA computation for counterparty risk assessment in credit portfolios. Credit Risk Frontiers, Bielecki, T.R., Brigo, D. and Patras, F., eds., Wiley/Bloomberg-Press, 2011.

[2] Basel Committee on Banking Supervision: Revisions to the Basel II market risk framework, December 2010.

[3] Bielecki, T.R., Crépey, S., Jeanblanc, M.: Up and down credit risk. Quantitative Finance 10 (10), pp. 1137-1151 (2010).

[4] Bielecki, T.R., Cousin, A., Crépey, S., Herbertsson, A.: Dynamic Hedging of Portfolio Credit Risk in a Markov Copula Model. Forthcoming in Journal of Optimization Theory and Applications.

[5] Bielecki, T.R., Cousin, A., Crépey, S., Herbertsson, A.: A Bottom-Up Dynamic Model of Portfolio Credit Risk - Part II: Common-shock interpretation, calibration and hedging issues (submitted).

[6] Bielecki, T.R., Cousin, A., Crépey, S., Herbertsson, A.: A bottom-up dynamic model of portfolio credit risk with stochastic intensities and random recoveries (submitted). 
[7] Bielecki, T. R. And Crépey, S.: Dynamic Hedging of Counterparty Exposure. Forthcoming in The Musiela Festschrift, Zariphopoulou, T., Rutkowski, M. and Kabanov, Y., eds, Springer.

[8] Bielecki, T.R., Crépey, S., Jeanblanc, M. and Zargari, B.: Valuation and Hedging of CDS Counterparty Exposure in a Markov Copula Model. Forthcoming in International Journal of Theoretical and Applied Finance.

[9] Bielecki, T. R. and Jakubowski, J. And Niewȩglowski, M.: Dynamic Modeling of Dependence in Finance via Copulae Between Stochastic Processes, Copula Theory and Its Applications, Lecture Notes in Statistics, Vol.198, Part 1, 33-76, 2010.

[10] Bielecki, T.R., Vidozzi, A. And Vidozzi, L.: Collateralized CVA Valuation with Rating Triggers and Credit Migrations, J. of Credit Risk, submitted, 2012.

[11] Bielecki, T.R., Cialenco, I. And Iyigunler, I.: A Markov Copulae Approach to Pricing and Hedging of Credit Index Derivatives and Ratings Triggered Step-Up Bonds, J. of Credit Risk, 2008.

[12] Brigo, D., Pallavicini, A., Torresetti, R.: Calibration of CDO Tranches with the dynamical Generalized-Poisson Loss model. Risk Magazine, May 2007.

[13] Brigo, D., Pallavicini, A., Torresetti, R. Credit models and the crisis: default cluster dynamics and the generalized Poisson loss model, Journal of Credit Risk, 6 (4), 39-81, 2010.

[14] Brigo, D., Pallavicini, A., Torresetti, R. Cluster-based extension of the generalized poisson loss dynamics and consistency with single names. International Journal of Theoretical and Applied Finance, Vol 10, n. 4, 607-632, 2007.

[15] Cont, R. And Kan, Y.H.: Dynamic Hedging of Portfolio Credit Derivatives, SIAM Journal on Financial Mathematics, 2, p. 112-140, 2011.

[16] Cont, R., Minca, A.: Recovering Portfolio Default Intensities Implied by CDO Quotes, Working Paper, 2008.

[17] Cousin, A., CréPey, S. And Kan, Y.H.: Delta-hedging Correlation Risk? Review of Derivatives Research, June 2011.

[18] CrÉPey, S.: Financial Modeling: A Backward Stochastic Differential Equations Perspective, Springer, 2013 (forthcoming).

[19] Crépey, S. and Rahal, A.: Simulation/Regression Pricing Schemes for CVA Computations on CDO Tranches (submitted).

[20] Duffie, D. And GÂrleanu, N.: Risk and the valuation of collateralized debt obligations, Financial Analysts Journal, 57, 41-62, 2001.

[21] Elouerkhaoui, Y.: Pricing and Hedging in a Dynamic Credit Model. International Journal of Theoretical and Applied Finance, Vol. 10, Issue 4, 703-731, 2007.

[22] Ethier, H.J. And Kurtz, T.G.: Markov Processes. Characterization and Convergence. Wiley, 1986. 
[23] Frey, R., Backhaus, J. Dynamic hedging of synthetic CDO tranches with spreadand contagion risk, Journal of Economic Dynamics and Control, 34 (4), 710-724, 2010.

[24] Herbertsson, A. (2011) Modelling default contagion using Multivariate Phase-Type distributions, Review of Derivatives Research 14 (1), 1-36.

[25] Herbertsson, A. And Rootzén, H. (2008) Pricing kth-to-default swaps under default contagion:the matrix-analytic approach, The Journal of Computational Finance $12(1), 49-78$.

[26] JACOD, J.: Calcul Stochastique et Problèmes de Martingales. Springer, 2nd edition, 2003.

[27] Jeanblanc, M., Yor, M. And Chesney, M.: Mathematical methods for Financial Markets. Springer, 2009.

[28] Laurent, J.-P., Cousin, A. And Fermanian, J-D.: Hedging default risks of CDOs in Markovian contagion models. Quantitative Finance, 1469-7696, April 2010.

[29] Lindskog, F. And McNeil, A. J.: Common Poisson shock models: applications to insurance and credit risk modelling. ASTIN Bulletin, 33(2), 209-238, 2003

[30] Marshall, A. \& Olkin, I.: A multivariate exponential distribution, J. Amer. Statist. Assoc., 2, 84-98, 1967.

[31] Patton, A.: Modelling Time-varying exchange rate dependence using the conditional copula, Working Paper 2001-09, University of California, San Diego, 2001.

[32] Protter, P.E.: Stochastic Integration and Differential Equations, Second Edition, Version 2.1. Springer, 2005.

[33] Rogers, L.C.G. And Pitman J.W.: Markov functions, Ann. of Prob., 9, 578-582, 1981.

[34] Schweizer, M.: A Guided Tour through Quadratic Hedging Approaches. In Option Pricing, Interest Rates and Risk Management, E. Jouini, J. Cvitanic and M. Musiela, eds., Cambridge University Press, 538-574, 2001. 